\title{
Prevalence of rifampicin resistant tuberculosis and associated factors among presumptive tuberculosis patients in a secondary referral hospital in Lagos Nigeria
}

\author{
Olusola Adedeji Adejumo ${ }^{1}$, Bolanle Olusola-Faleye ${ }^{2}$, Victor Adepoju ${ }^{2}$, Abimbola Bowale ${ }^{1}$, Sunday Adesola ${ }^{1}$, \\ Ayodeji Falana ${ }^{1}$, Henry Owuna ${ }^{1}$, Kehinde Otemuyiwa ${ }^{1}$, Shafaatu Oladega ${ }^{1}$, Oluwatosin Adegboye ${ }^{2}$
}

1. Mainland Hospital, Yaba Lagos Nigeria.

2. KNCV TB Foundation Nigeria/Challenge TB project, Lagos Nigeria.

\begin{abstract}
Background: Nigeria is one of the 30 high burden countries for drug resistant tuberculosis (DR-TB). This study assessed the prevalence and factors associated with rifampicin resistant tuberculosis (RR-TB) in a secondary referral hospital in Lagos State Nigeria.

Methods: A retrospective review of presumptive TB register of patients screened for Mycobacterium tuberculosis (MTB) and RRTB using Xpert MTB/RIF assay at Mainland hospital between January 12012 and December 312016 was conducted.

Results: A total of 2497 clients were screened for MTB and RR-TB during the study period. The majority (51.4\%) were between 25 - 44 years. Male: Female ratio was 1:0.8. Of the 2497 clients screened, MTB was detected in 942 (37.7\%) out of which 220 (23.4\%) had RR-TB. Age (AOR 1.8, 95\%CI 1.3- 2.6, p = 0.001), symptomatic contact with DR-TB patients (AOR 3.3, 95\%CI 2.1-5.1, p <0.001) and type of TB (AOR 2.9, 95\% CI 1.7 - 5.0, <0.001) were associated with RR-TB after adjusting for age, gender, HIV status and symptomatic contacts with DR-TB patients.

Conclusion: The prevalence of RR-TB in new and previously treated TB patients was high in this study. Urgent steps are needed to avert an impending RR-TB epidemic

Keywords: Rifampicin resistance, tuberculosis, Xpert MTB/RIF, Lagos.

DOI: https://dx.doi.org/10.4314/ahs.v18i3.2

Cite as: Adejumo OA, Olusola-Faleye B, Adepoju V, Bowale A, Adesola S, Falana A, Owuna H, Otemuyiwa K, Oladega S, Adegboye O. Prevalence of rifampicin resistant tuberculosis and associated factors among presumptive tuberculosis patients in a secondary referral hospital in Lagos Nigeria. Afri Health Sci. 2018;18(3): 472-478. https:/ / dx.doi.org/10.4314/ahs.v18i3.2
\end{abstract}

\section{Introduction}

The emergence of multidrug-resistant tuberculosis (MDR-TB) defined as resistance to at least rifampicin and isoniazid and rifampicin-resistant TB (RR-TB) has been a major obstacle to TB control globally. ${ }^{1}$ A World Health

\section{Corresponding author: \\ Olusola Adedeji Adejumo, \\ Mainland Hospital Yaba Lagos Nigeria. \\ Email: oluadejumo75@gmail.com}

Organization (WHO) report indicated that 580,000 new cases of MDR/RR-TB were notified in 2015 and an estimated 250,000 MDR/RR-TB patients died in 2015. ${ }^{2}$ Surveillance data show that about $4 \%$ of new and $21 \%$ of previously treated TB cases had MDR/RR-TB in 2015. ${ }^{3}$ Although there has been an increase in TB and MDR/ RR-TB case notifications, the gaps in case detection and treatment remain due to under reporting (in countries with large private sector) and under diagnosis (due to physical and financial barriers). ${ }^{2}$

The TB burden in Nigeria is one of the highest in the world and Nigeria is one of the 14 countries on the high burden lists for TB, MDR-TB and TB/HIV. ${ }^{2}$ In addition, 
Nigeria is one of the six countries contributing $60 \%$ of the 10.4 million new cases in 2015 and one of the five countries that accounted for over $60 \%$ gap between estimated number of incident MDR/RR-TB cases and MDR-TB enrolment in 2015. ${ }^{2}$ A meta-analysis of the prevalence of drug resistant TB (DR-TB) in Nigeria indicated that $6 \%$ and $32 \%$ of newly and previously treated TB cases respectively had MDR-TB. ${ }^{4}$

Gene Xpert ${ }^{\circledR}$ MTB/RIF (Xpert) (Cepheid Inc. New Jersey, USA) for Mycobacterium tuberculosis (MTB) detection was endorsed by the WHO in $2010 \cdot{ }^{5-7}$ It offers a platform for the integration of TB and HIV activities in high burdened TB and HIV settings ${ }^{8}$. The Xpert MTB/RIF is a point of care automated diagnostic test that can identify the DNA of Mycobacterium tuberculosis (MTB) and rifampicin resistance. It has high sensitivity and specificity at $97.6 \%$ and $99.8 \%$ respectively. ${ }^{9}$

The use of Xpert MTB/RIF assay commenced in Nigeria in 2012 and the programme was implemented by the KNCV TB Foundation in partnership with the Nigerian government, the support of the United States Agency for International Development (USAID) and the supervision of the National TB Programme (NTP). ${ }^{10}$ The use of Xpert MTB/RIF assay by the Lagos State TB and Leprosy Control Programme (LSTBLCP) started in 2012. At inception, the Xpert assay was limited to priority groups which includes known symptomatic contacts of DR-TB for adults and all child contacts of DR-TB patients, all previously treated drug susceptible TB cases (treatment failure, relapse, treatment after loss to follow up and other previously treated patients) and TB/HIV co-infected patients. However, since 2016 the NTP recommended Xpert MTB/RIF assay for all presumptive TB patients in centers where it is available. ${ }^{11}$ This present study assessed the prevalence and factors associated with RR-TB in a secondary referral hospital in Lagos State Nigeria.

\section{Methods \\ Study design}

A retrospective review of presumptive TB register of patients screened for Mycobacterium tuberculosis (MTB) and RR-TB using Xpert MTB/RIF assay at Mainland hospital between January 12012 and December 312016 was conducted.

\section{Study background}

This study was conducted at Mainland Hospital in Lagos state Nigeria. Mainland Hospital is a secondary referral facility established in 1930 as a sanitorium. Formally known as Infectious Disease Hospital (IDH), the name was changed to Mainland Hospital in 2003. The hospital is a referral hospital for treatment of infectious diseases like TB, HIV/AIDS, MDR-TB, Cholera, Ebola virus disease and Lassa fever in Lagos state and its environ. The bed capacity of the hospital is 100 out of which 40 was designated for MDR-TB patients. The MDR-TB complex (the only one in the state) was commissioned 2012 by the Lagos State Government in collaboration with USAID. That same year, the programmatic management of DR-TB patients commenced in the hospital. MDRTB patients were admitted for the entire duration of the intensive phase (8 months), however treatment during the continuation phase was done on out-patient basis. In 2014, community MDR-TB management commenced; this involved treatment of MDR-TB patients in the community. Health workers were assigned to MDR-TB patients to monitor treatment and administer the injections. Lagos state is located in SouthWest Nigeria with the estimated population of $12,155,337$ in $2015 .{ }^{12}$ Although Lagos State is one of the smallest state in the country, it is the commercial nerve center of Nigeria. About 11\% of Nigerian population live in Lagos state and it has a total of 20 LGAs and 37 local council development areas (LCDAs)

\section{Programmatic Management of Drug Resistant TB in Lagos State}

The LSTBLCP is responsible for the coordination of TB control activities in Lagos state. All presumptive TB patients are expected to do Xpert MTB/RIF assay in the state to determine their bacteriological status and resistance to rifampicin. Patients with positive MTB and rifampicin resistance were sent for Line Probe Assay (LPA) and Culture. Other baseline investigations like electrolytes and urea, creatinine, urinalysis, full blood count, liver function test, hepatitis B surface antigen, chest X-ray, thyroid function test, audiometry and HIV tests were also conducted.

Drug management of MDR/RR-TB patients is for 20 months divided into 8 months intensive phase and 12 
months continuation phase. Levofloxacin, Cycloserine, Prothinamide, Kanamycin/ Capromycin, Pyrazinamide and Pyridoxine were drugs used during the intensive phase. Kanamycin or Capromycin is excluded in the continuation phase. HIV counseling and testing was offered to all presumptive TB patients. Patients with HIV co-infection were offered anti-retroviral drugs two weeks after DR-TB treatment initiation if they were not previously on anti-retrovirals.

\section{Data analysis}

Statistical Package for Social Sciences (SPSS) IBM version 22 was used for data analysis. Numerical variables were presented in percentages, mean and standard deviation. Categorical variables were compared using chi squared test. Rifampicin resistance was the outcome variable. Crude and adjusted odds ratio of associated factors of the outcome variable (age, gender, HIV status, symptomatic contact with MDR patients and type of TB) were determined. Confidence interval was set at 95\% and $\mathrm{p}$
$<0.05$ was considered significant for all statistical tests.

\section{Ethical issues}

Permission to use data was obtained from the management of Mainland hospital. Data was de-identified before use.

\section{Results}

A total of 2497 client were screened for MTB and RRTB during the study period. The majority $(51.4 \%)$ were between 25 - 44 years. There were more males $(55 \%)$ than females (45\%). About 35\% had HIV co-infection. Of the 2497 clients screened, MTB was detected in 942 (37.7\%) while 166 (6.6\%) were invalid. Among the MTB cases found, $220(23.4 \%)$ had RR-TB as shown in Table 1. The proportion of clients screened for MTB and RR- TB increased from $11 \%$ in 2012 to $32.9 \%$ in 2016. However, the proportion of MTB positive cases detected declined from $49.3 \%$ in 2012 to $38.4 \%$ in 2016 . In addition, the proportion of RR-TB cases detected reduced from $48.9 \%$ in 2012 to $13 \%$ in 2016 (Table 1).

Table 1: Characteristics of presumptive TB clients seen between 2012 and 2016

\begin{tabular}{|c|c|c|c|c|c|c|c|}
\hline Variables & $\begin{array}{l}2012 \\
n=274 \\
(\%)\end{array}$ & $\begin{array}{l}2013 \\
n=400(\%)\end{array}$ & $\begin{array}{l}2014 \\
n=496 \\
(\%)\end{array}$ & $\begin{array}{l}2015 \\
n=505(\%)\end{array}$ & $\begin{array}{l}2016 \\
n=822(\%)\end{array}$ & $\begin{array}{l}\text { Total } \\
n=2497(\%)\end{array}$ & \\
\hline \multicolumn{8}{|l|}{ Age group } \\
\hline $0-14$ & $4(1.5)$ & $8(2.0)$ & $12(2.4)$ & $11(2.2)$ & $12(1.5)$ & & $47(1.9)$ \\
\hline $15-24$ & 45 (16.4) & $60(15.0)$ & $58(11.7)$ & 61 (12.1) & 93 (11.3) & & $317(12.7)$ \\
\hline $25-34$ & $70(25.5)$ & $100(25.0)$ & $\begin{array}{r}108 \\
(21.8)\end{array}$ & 169 (33.5) & 180 (21.9) & & $627(25.1)$ \\
\hline $35-44$ & $68(24.8)$ & $106(26.5)$ & $\begin{array}{r}127 \\
(25.6)\end{array}$ & $107(21.2)$ & 249 (30.3) & & $657(26.3)$ \\
\hline $45-54$ & $51(18.6)$ & $80(20.0)$ & $\begin{array}{r}110 \\
(22.2)\end{array}$ & $48(9.5)$ & $176(21.4)$ & & 465 (18.6) \\
\hline $55-64$ & $24(8.8)$ & $32(8.0)$ & $50(10.1)$ & 55 (10.9) & $83(10.1)$ & & $244(9.8)$ \\
\hline$\geq 65$ & $12(4.4)$ & $14(3.5)$ & $31(6.3)$ & $54(10.7)$ & $29(3.5)$ & & $140(5.6)$ \\
\hline \multicolumn{8}{|l|}{ Gender } \\
\hline Male & $154(56.2)$ & $229(57.3)$ & $\begin{array}{r}277 \\
(55.8)\end{array}$ & 247 (48.9) & 466 (56.7) & & $1373(55.0)$ \\
\hline Female & $120(43.8)$ & $171(42.8)$ & $\begin{array}{r}219 \\
(44.2)\end{array}$ & $258(51.1)$ & $356(43.3)$ & & $1124(45.0)$ \\
\hline \multicolumn{8}{|l|}{ HIV result } \\
\hline Positive & $33(12.0)$ & $156(39.0)$ & $\begin{array}{r}160 \\
(32.3)\end{array}$ & 200 (39.6) & $312(38.0)$ & & $861(34.5)$ \\
\hline Negative & $161(58.8)$ & $161(40.3)$ & $\begin{array}{r}320 \\
(64.5)\end{array}$ & $282(55.8)$ & $487(59.2)$ & & $1411(56.5)$ \\
\hline Unknown & $80(29.2)$ & $83(20.8)$ & $16(3.2)$ & $23(4.6)$ & $23(2.8)$ & & 225 (9.0) \\
\hline \multicolumn{8}{|c|}{ MTB detection } \\
\hline Negative & $118(43.1)$ & $170(42.5)$ & $\begin{array}{r}274 \\
(55.2)\end{array}$ & $388(76.8)$ & $439(53.4)$ & & $1389(55.6)$ \\
\hline Positive & 135 (49.3) & $181(45.3)$ & $\begin{array}{r}216 \\
(43.5)\end{array}$ & 94 (18.6) & $316(38.4)$ & & $942(37.7)$ \\
\hline invalid & $21(7.7)$ & $49(12.3)$ & $6(1.2)$ & $23(4.6)$ & $67(8.2)$ & & $166(6.6)$ \\
\hline $\begin{array}{l}\text { Rifampicin } \\
\text { resistance }\end{array}$ & $n=135(\%)$ & $\mathrm{n}=181(\%)$ & $\begin{array}{l}n=216 \\
(\%)\end{array}$ & $n=94(\%)$ & $\mathrm{n}=316(\%)$ & $n=942(\%)$ & \\
\hline No & $69(51.1)$ & $124(68.5)$ & $\begin{array}{r}182 \\
(82.3)\end{array}$ & $72(76.6)$ & 275 (87.0) & & $722(76.6)$ \\
\hline Yes & 66 (48.9) & 57 (31.5) & 34 (15.7) & $22(23.4)$ & $41(13.0)$ & & $220(23.4)$ \\
\hline
\end{tabular}


Figure 1 shows the indication for Xpert MTB/RIF assay. The majority $(43.3 \%)$ were screened for RR-TB because of symptomatic contact with DR-TB patients. Other rea- sons for Xpert MTB/RIF assay includes PLHIV with symptoms of TB (21.7\%), TB relapse (13.4\%) and failed six months regimen (3.2\%). The regression analysis of the factors associated with RR-TB is shown in Table 2.

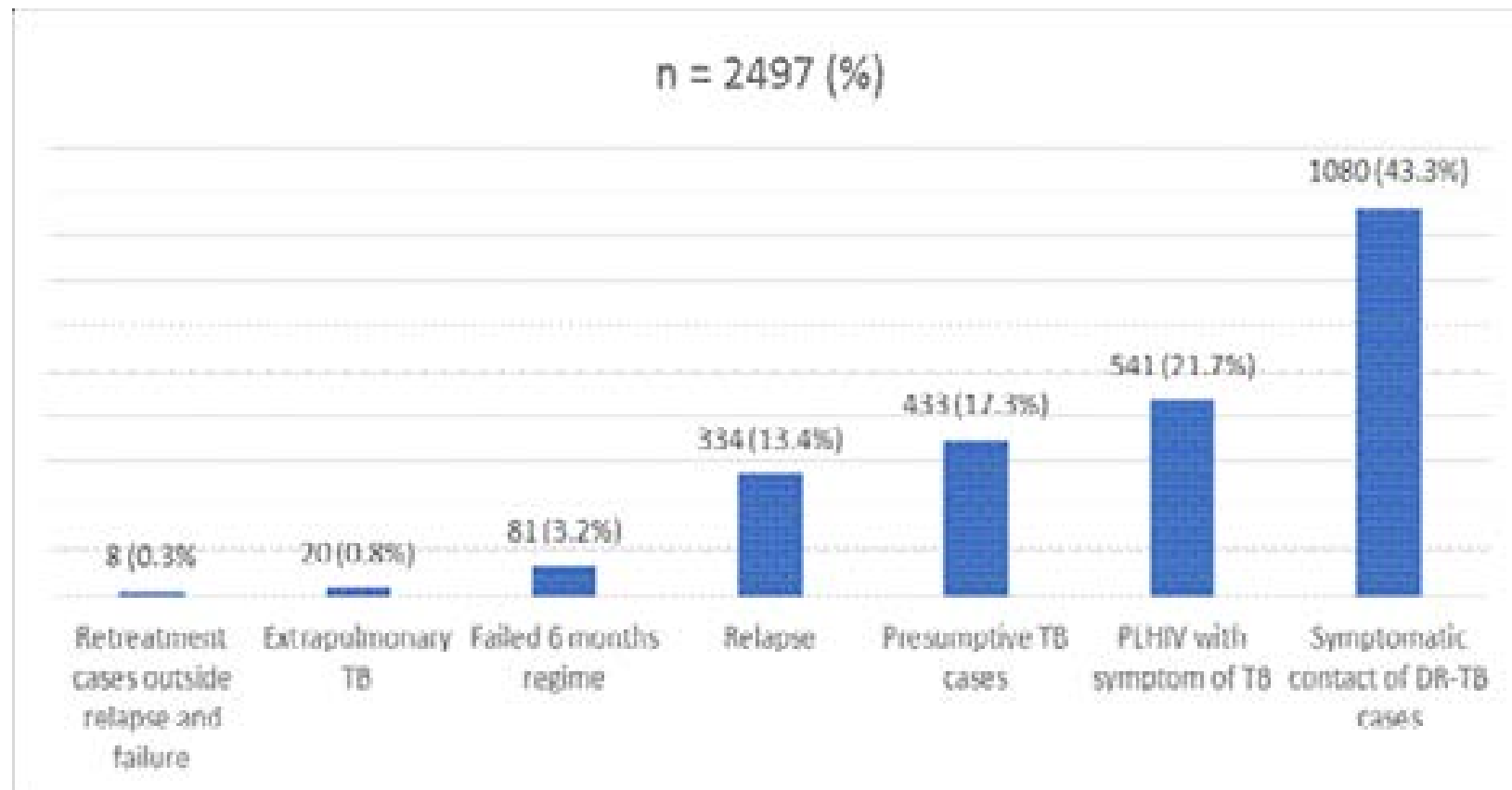

Figure 1: Reasons for Xpert MTB/RIF assay 
Table 2: Regression analysis of factors associated with DR-TB

\begin{tabular}{|c|c|c|c|c|}
\hline Variable & MDR/RIF Positive & MDR/RIF Negative & $\operatorname{COR}(95 \% \mathrm{CI}), \mathrm{p}$ & $\operatorname{AOR}(95 \% \mathrm{CI}), \mathrm{p}$ \\
\hline \multicolumn{5}{|l|}{ Age group (years) } \\
\hline$<45$ & $137(20.2)$ & $541(79.8)$ & 1 & 1 \\
\hline$\geq 45$ & $83(31.4)$ & $181(68.6)$ & $1.8(1.3-2.5),<0.001$ & $1.8(1.3-2.6), \mathbf{0 . 0 0 1}$ \\
\hline \multicolumn{5}{|l|}{ Gender } \\
\hline Male & $148(25.9)$ & $424(74.1)$ & $1.4(1.0-2.0), 0.023$ & $1.4(0.9-1.9), 0.099$ \\
\hline Female & $72(19.5)$ & $298(80.5)$ & 1 & 1 \\
\hline \multicolumn{5}{|l|}{ HIV status } \\
\hline Negative & $142(22.2)$ & $497(77.8)$ & $1.03(0.7-1.56), 0.866$ & $1.2(0.8-1.9), 0.280$ \\
\hline Positive & $42(21.6)$ & $152(78.4)$ & 1 & \multirow[t]{2}{*}{1} \\
\hline Unknown ${ }^{\#}$ & $36(33.0)$ & $73(67.0)$ & & \\
\hline \multicolumn{5}{|c|}{ Symptomatic contact with } \\
\hline \multicolumn{5}{|c|}{ MDR patient } \\
\hline Yes & $141(29.2)$ & $342(70.8)$ & $2.0(1.4-2.7),<0.001$ & \multirow[t]{2}{*}{$3.3(2.1-5.1),<0.001$} \\
\hline No & $79(17.2)$ & $380(82.8)$ & 1 & \\
\hline \multicolumn{5}{|l|}{ Type of TB } \\
\hline Retreatment cases & $51(26.7)$ & $140(73.3)$ & $1.25(0.86-1.83), 0.220$ & $2.9(1.7-5.0)$ \\
\hline New & $169(22.5)$ & $582(77.5)$ & 1 & 1 \\
\hline
\end{tabular}

Age, symptomatic contact with DR-TB patients and type of TB were factors associated with RR-TB. Significantly higher proportion of TB patients aged 45 years and older $(31.4 \%)$ had rifampicin resistance compared with patients below 45 years (20.2\%) AOR 1.8 (95\%CI 1.3- 2.6) $\mathrm{p}=0.001$. The odds for symptomatic contact of DRTB patients to develop rifampicin resistance was about three-fold higher than patients without symptomatic contact with DR-TB patients AOR 3.3, (95\%CI 2.1-5.1), $\mathrm{p}<0.001$. The proportion of RR-TB among previously treated TB patients was higher $(26.7 \%)$ than among newly diagnosed TB patients $(22.5 \%)$ and the chance of developing RR-TB was three times more in patients previously treated for TB AOR $2.9(95 \%$ CI 1.7 - 5.0) after adjusting for age, gender, HIV status and symptomatic contacts with DR-TB patients.

\section{Discussion}

The prevalence of RR-TB was $23.4 \%$ in this study $(22.5 \%$ and $26.7 \%$ among new and previously treated TB patients respectively). This finding is high compared to what was reported in studies from Southern and Northern part of Nigeria, Ethiopia and Burundi. ${ }^{13-16}$ However, studies from another Southern state in Nigeria indicated a higher prevalence than what was found in this study. ${ }^{17} \mathrm{~A}$ sys- tematic review of the prevalence of DR-TB in Nigeria showed that the prevalence of DR-TB in the country varied across geographical regions, settings and the methods used. ${ }^{4}$ The high prevalence obtained in our study may be due to the study setting. Mainland hospital is a referral hospital in Lagos State for TB and MDR-TB. Presumptive DR-TB patients were referred from other hospitals in the state for Xpert MTB/RIF. The reducing trend in the prevalence of DR-TB in this study may be due to the increase in the number of Xpert MTB/RIF sites in the state. The Xpert MTB/RIF sites in Lagos increased from 7 in 2013 to 27 in 2016. In addition, there was a change in policy regarding the use of Xpert MTB/RIF within the study period. Xpert MTB/RIF assay which was initially limited to priority groups such as TB/HIV co-infected patients, symptomatic contact of DR-TB adults and previously treated drug sensitive TB patients was recommended for all presumptive TB patients by the NTP. ${ }^{11}$

Findings from systematic review on the prevalence of DR-TB in Nigeria and Ethiopia ${ }^{4,18}$ have shown that the current WHO estimate for new and previously treated TB patients for both countries were low which is proof that the burden of DR-TB in Nigeria and other high TB burden countries in Africa is probably bigger than we anticipated. $^{2}$ 
Many studies have demonstrated that history of previous TB treatment is a strong risk factor for MDR-TB. ${ }^{19-22}$ This could be due to poor quality of anti-TB drugs used, repeated, inappropriate intake of medications and poor management of drug sensitive TB. A survey which analyzed data from 11 countries indicated that the chance of developing resistant TB increased with the length of time of exposure to anti-TB drugs. ${ }^{23}$ In this study, the chance of developing RR-TB was about three fold higher among patients previously treated for TB than new TB patients. Before the introduction of Xpert MTB/RIF assay, the NTP recommended the addition of streptomycin to the anti-TB regimen of previously treated TB patients which prolonged the exposure and consequent resistance to anti-TB drugs.

In this study, the chance of developing RR-TB was higher among patients with symptomatic contact with a known DR-TB patient than those without contact. This finding underscores the continual spread of DR-TB in the community mostly due to failure to promptly diagnose presumptive DR-TB patient and early treatment initiation. A WHO report showed that of the 1241 MDR/RR-TB cases diagnosed in 2015, about 50\% were enrolled for treatment. In addition, Nigeria is one of the five countries responsible for over $60 \%$ gap between incident MDR/ RR-TB cases and treatment enrolment ${ }^{2}$.

The chance of RR-TB was about two-fold higher among patients aged 45 years and above than the younger age group in this study similar to what was reported in a study from Northern Nigeria. ${ }^{24}$ The association between age and MDR-TB is not consistent. While studies form Nigeria, South Africa and Ethiopia have demonstrated a higher prevalence of DR- TB in the lower age groups, ${ }^{13,25-27}$ another Nigerian study showed no significant difference between age and DR-TB. The population structure of the study settings may account for the inconsistent findings. However, surveillance data from Central and Eastern Europe have shown that prevalence of MDR-TB peaks in young adulthood. ${ }^{27}$

There was no gender difference in the prevalence of RRTB in this study similar to what was reported from other Nigerian studies. ${ }^{13,22,25}$ Studies from the Northern and Southern part of Nigeria and South Africa showed that the prevalence of DR-TB was significantly higher among males. ${ }^{14,24,26,28}$ Poor health seeking behavior among men, social stigma and cultural habits were adduced for the high prevalence of DR-TB among men. ${ }^{28}$

\section{Limitation}

The issue of missing and incomplete data is a major limitation of this study like any other retrospective survey. Available complete data was used for analysis and estimation of the RR-TB prevalence.

\section{Conclusion}

The prevalence of RR-TB in new and previously treated TB patients was high in this study. Reduction of the prevalence of MDR/RR-TB is a daunting task. Proactive measures are needed urgently to address the challenge of prompt diagnosis and early initiation of DR-TB treatment to reduce spread of DR-TB in the community. In addition, the LSTBLCP must put mechanisms in place to ensure adequate treatment of drug sensitive TB.

\section{Conflict of interest}

All authors declare no conflict of interest.

\section{References}

1. Burki T. Tuberculosis-resistance, funding, and drugs. Lancet Infect. 2010; Dis 10: 297-298.

2. World Health Organization, Global Tuberculosis Report 2016, World Health Organization, Geneva, Switzerland, 2016. Available from www.who.int/tb/publications/ global_report/en/ (Last accessed September 2017).

3. Falzon D, Mirzayev F, Wares F, Baena IG, Zignol M, Linh N, et al. Multidrug-resistant tuberculosis around the world: what progress has been made? Eur Respir J. 2015; 45(1):150-160.

4. Onyedum CC, Alobu I, Ukwaja KN. Prevalence of drug-resistant tuberculosis in Nigeria: A systematic review and meta-analysis. PLoS ONE 2017;12(7): e0180996. PubMed. https://doi.org/ 10.1371/journal. pone.0180996

5. Cresswell J, Codlin AJ, Andre E. Results from early programmatic implementation of Xpert MTB/RIF testing in nine countries. BMC Infect Dis. 2014; 14: 2.

6. VanRie A, Page-Ship L, Scott L, Sanne I, Stevens W. Xpert MTB/ RIF for point-of-care diagnosis of tuberculosis in high HIV burden, resource-limited countries; Hype or hope. Expert Rev Mol Diagn. 2010;10: 937-946.

7. Dereje AG, Yoseph CM, Adugua NG, Gizachew TA, 
Melaku TD, Kassu DT. Xpert MTB/RIF assay for diagnosis of pulmonary tuberculosis in sputum specimens in Remote healthcare facilities. BMC Microbiol. 2015;15: 220. doi: 10.1186/s12866-015-0566-6

8. Narasimooloo R, Ross A. Delay in commencing treatment for MDR TB at a specialised TB treatment centre in KwaZulu-Natal. S Afr Med J. 2012;102:360- 362.

9. Boehme CC, Nabeta P, Hillemann D, Nicol MP, Shenai S, Krapp F et al. Rapid molecular detection of tuberculosis and rifampin resistance. N EnglJ Med. 2010; 363:10051015.

10. Gidado M, Onazi OO, Nwadike P, Emeka E, Akang G, Eneogu R, et al. Assessment of gene-Xpert MTB RIF program implementation and the challenges for enhanced tuberculosis diagnosis in Nigeria. SAARCJ Tuber Lung Dis HIV/AIDS. 2015; 12: 1-7. PubMed .

11. Federal Ministry of Health. National Tuberculosis and Leprosy Control programme. Module for training doctors, nurses, state team and consilium of experts on the clinicaland programmatic management of drug resistant tuberculosis $2^{\text {nd }}$ edition Abuja.

12. National Bureau of Statistics. Available fromhttp:// www.nigerianstat.gov.ng/(Last accessed September 2017) 13. Kuyinu YA, Odugbemi BA, Salisu-Olatunji SO, Adepoju FO, Odusanya OO. Characteristics of Mycobacterium Tuberculosis Positive Patients Screened for Drug-Resistant Tuberculosis at a Tertiary Health Facility in Lagos, J Natl Med Assoc. 2018:110:89-91. PubMed .

14. Audu ES, Gambo MS, Yakubu AA. Rifampicin resistant mycobacterium tuberculosis in Nasarawa State, Nigeria. Niger J Basic Clin Sci. 2017;14:21-25.

15. Adane K, Ameni G, Bekele S, Abebe M, Aseffa A. Prevalence and drug resistance profile of mycobacterium tuberculosis isolated from pulmonary tuberculosis patients attending two public hospitals in East Gojjam zone, northwest Ethiopia. BMC Public Health. 2015; 15(1), 572 PubMed.

16. Sanders M, Van Deun A, Ntakirutimana D, Masabo JP, Rukundo J, Rigouts L, et al. Rifampicin monoresistant Mycobacterium tuberculosis in Bujumbura, Burundi: results of a drug resistance survey. Int J Tuberc Lung Dis. 2006; 10: 178 183 . PMID: 16499257

17. Osman E, Daniel O, Ogiri S, Awe A, Obasanya O, Adebiyi E et al. (2012) Resistance of Mycobacterium Tuberculosis to First and Second Line Anti Tuberculosis
Drugs in South West, Nigeria. J Pulmon Resp Med 2012; doi:10.4172/2161-105X.S6-001.

18. Weldegebreal S, Mebrahtu T. Anti-tuberculosis drug resistance in Ethiopia: systematic review. Int J Tuberc Lung Dis. 2017; 21:18-22 .

19. Gunther G, Van Leth F, Alexandru S, Altet N, Avsar $\mathrm{K}$, Bang D, et al. Multidrug-resistant tuberculosis in Europe, 2010-2011. Emerg Infect Dis. 2015;21:409-416.

20. Fregona G, Cosme LB, Moreira CMM, Bussular JL, Dettoni VV, Dalcolmo MP, et al. Risk factors associated with multidrug-resistant tuberculosis in Espirito Santo, Brazil. Rev Saude Publica. 2017;51:41.

21. Chuchottaworn C, Thanachartwet V, Sangsayunh P, Than TZM, Sahassananda D, Surabotsophon M, et al. Risk Factors for Multidrug-Resistant Tuberculosis among Patients with Pulmonary Tuberculosis at the Central Chest Institute of Thailand. PLOS ONE 2015;10(10): e0139986. PubMed. doi:10.1371/journal.pone.0139986.

22. Daniel OJ, Osman E, Prevalence and risk factors associated with drug resistant TB in SouthWest, Nigeria. Asian Pac J Trop Med. 2011;4:148-151.

23. Espinal MA, Laserson K, Camacho M, Fusheng Z, Kim SJ, Tlali. et al. Determinants of drug-resistant tuberculosis: analysis of 11 countries. Int J Tuberc Lung Dis. 2001;5(10): 887-893.

24. Tilako BH, Zakari B, Sado F, Yerima IB. Multi-drug resistance tuberculosis (MDR-TB) survey in North East. Nig J Pharm Cos Sci. 2013;1(6): 45-52. PubMed.

25. Lawson L, Yassin MA, Abdurrahman ST, Parry CM, Dacombe R, Sogaolu OM, et al. Resistance to first-line tuberculosis drugs in three cities of Nigeria. Trop Med Int Health. 2011; 16 (8): 974-980. PubMed.

26. Coovadia YM, Mahomed S, Pillay M, Werner L, Mlisana K. Rifampicin Mono Resistance in Mycobacterium tuberculosis in Kwazulu Natal, South Africa: A significant phenomenon in high prevalence TB/HIV Region. PLos One. 2013;8:e77712.

27. Workicho A, Kassahun W, Alemseged F. Risk factors for multidrug-resistant tuberculosis among tuberculosis patients: a case-control study. Infect Drug Resis. 2017;10: 91-96. PubMed.

28. Uzoewulu NG, Ibeh IN, Lawson L, Goyal M, Umenyonu N, Ofiaeli RO, et al.Drug Resistant Mycobacterium tuberculosis in Tertiary Hospital South East, Nigeria. J Med Microb Diagn. 2014, 3:2 http://dx.doi.org/10.4172/21610703.1000141

African Health Sciences Vol 18 Issue 3, September, 2018 